\title{
La UNIVERSIDAd (Des)CONOCida de Roberto Bolaño
}

\section{La Universidad (Des)conocida of Roberto Bolaño}

\author{
ALEXIS CANDIA CÁCERES \\ UNIVERSIDAD DE PLAYA ANCHA \\ ivan.candia@upla.cl
}

\begin{abstract}
Resumen: En el artículo se realiza un estudio de las conexiones de la literatura de Roberto Bolaño con algunos de los autores, textos y corrientes literarias más relevantes del canon occidental, tales como el romanticismo, las vanguardias, el simbolismo y la literatura de Jorge Luis Borges. Se busca demostrar que Roberto Bolaño no sólo dialoga con la tradición occidental sino que malinterpreta y subsume la tradición con el fin de dotar de un giro extraño a su producción literaria.
\end{abstract}

Palabras clave: Roberto Bolaño, intertextualidad, canon occidental

\begin{abstract}
Roberto Bolaño's literature with some of the authors, texts and relevant literary currents of the Western canon, such as Romanticism, vanguards, symbolism and literature of Jorge Luis Borges. It is quite evident that Roberto Bolaño not only speaks to the Western tradition but also subsumes and misunderstood it, all that in order to provide a strange twist to his literary output.
\end{abstract}

Keywords: Roberto Bolaño, intertextuality, western canon 
La universidad desconocida es el título del libro que recoge parte importante de la poesía de Roberto Bolaño, así como una imagen externa que refiere a estructuras internas del proceso creativo individual de cada escritor, que el autor de 2666 utiliza, continuamente, para referirse a la entidad responsable de la formación del "poeta", tal como la reconoce, por ejemplo, en la contraportada del texto que reúne su summa poética: "Creo que en la formación de todo escritor hay una universidad desconocida que guía sus pasos, la cual, evidentemente, no tiene sede fija, es una universidad móvil, pero común a todos". Al tratarse de la formación del escritor, es claro que buena parte de la acción de la universidad desconocida pasa por el contacto y construcción de la biblioteca, la que constituye la patria misma de un autor según Bolaño. La biblioteca es la serie de libros que marca y establece el itinerario así como las influencias y tendencias en la carrera literaria de un escritor. De cierta forma, la universidad desconocida traza, a través de una serie de juegos de ecos y rechazos con la tradición y la vanguardia, la ruta que ha de adoptar el narrador, el poeta o el cronista. Reconstruir las huellas, las marcas $y$, a su vez, las reapropiaciones $y$ remodelaciones de la tradición operadas por Bolaño, constituye el núcleo central de este artículo.

Bajo esta lógica, apunto a revelar las conexiones que la obra bolañiana establece con algunos de los autores y textos canónicos de la tradición occidental. No me interesa solo archivar las influencias que reciben los textos de Bolaño, sino explorar la forma en que enfrentan la tradición y desvían la norma a fin de lograr la originalidad que le permita aspirar a la "pervivencia literaria". Concepto que, en el piélago de paradojas y antinomias por los que Bolaño desarticula las categorías absolutas, es simultáneamente deseado y menospreciado por el autor de Estrella distante, tal como plantea Celina Manzoni en "Ficción de futuro y lucha por el canon en la narrativa de Roberto Bolaño".

Así, me interesa construir un par de breves análisis comparativos entre los textos de Bolaño y ciertos autores y obras fundamentales de la literatura occidental, que permitan, por cierto, apreciar el proceso de construcción y reconstrucción del canon propuesto por el proyecto escritural de Roberto Bolaño.

Hablo de literatura occidental y no mundial porque pienso que la literatura bolañiana tiene, a todas luces, voluntad de ocaso. De hecho, Bolaño adopta y asimila la influencia de parte importante del canon occidental. Lo anterior, se evidencia al considerar el amplio abanico de escritores que influye en su obra, tal como reconoce en una entrevista con Carmen Boullosa:

Aldana, Manrique, Cervantes, los cronistas de Indias, Sor Juana, Fray Servando, Teresa de Mier, Pedro Henríquez Ureña, Rubén Darío, Alfonso Reyes, Borges [...] Me interesa la literatura francesa, la lucha de Pascal, que siempre intuyó su muerte, contra la melancolía [...] 0 
la ingenuidad adánica de Fourier. O toda esa prosa, generalmente anónima, de autores galantes, mitad costumbristas y mitad anatomistas y que, de algún modo, desemboca en la caverna interminable que es el marqués de Sade. También me interesa la literatura norteamericana del ochocientos, sobre todo Twain $y$ Melville, y la poesía de Emily Dickinson y Whitman. Cuando era adolescente hubo una época en que sólo leía a Poe. (Boullosa, 2002: 110)

Los estudios críticos que han abordado los ecos y reflejos de la universidad desconocida de Roberto Bolaño lo sitúan, también, en relación con la tradición occidental. Wilfrido Corral propone que la prosa bolañiana está bajo el influjo de ciertos autores canónicos europeos: "[...] decir que podría ser nuestro Joyce y Kafka. Veo, también, que Waugh, Amis (padre e hijo), una pizca de Nabokov, Philip Roth y más Perec que Houellebecq" (Corral, 2004: 36). Para Gonzalo Aguilar resulta clave, en tanto, la ascendencia de ciertos autores argentinos, "[...] se reconocen en su escritura la presencia de J.R. Wilcock, Jorge Luis Borges, Ricardo Piglia y H. Bustos Domecq" (Aguilar, 2002: 148). En Fuera de quicio, Villalobos sostiene que Bolaño era un autor autodidacta:

[...] que buscaba abismos y extremos, pero lo que llama nuestra atención es la reciente celebración de su figura como uno de los más importantes escritores de la actual renovación de la tradición literaria occidental: no se trata aún de un clásico, pero sí "del más prominente miembro de su generación"; en pocas palabras, de un nuevo maestro. (Villalobos, 2012: 194)

Roberto Bolaño lee y recibe las influencias de la literatura clásica (Homero, Esquilo, Virgilio), la literatura medieval (Geoffrey Chaucer, Boccaccio, Cantares de Gesta), española (Cervantes), francesa (Rimbaud, Breton, Camus, Perec), alemana (Goethe, Schlegel, Kafka, Jünger, Boll), inglesa (Shakespeare, Joyce, Woolf), italiana (Dante), rusa (Dostoievski), norteamericana (Poe, Melville, Twain, Foulkner, Kerouac, Pynchon), hispanoamericana (Maples Arce, Parra, Lihn, Borges, Cortázar, Fernández, García Márquez), por citar algunos.

La lectura de los libros de Roberto Bolaño evidencia, una y otra vez, el deseo de eliminar las barreras entre el arte y la realidad, tendencia que el autor de Los detectives salvajes establece desde sus primeras incursiones en el mundo de las letras. Es más, en "Déjenlo todo, nuevamente. Primer manifiesto infrarrealista", firmado por el propio Bolaño, establece que: "Nuestra ética es la Revolución, nuestra estética la Vida: una-sola-cosa" (Bolaño, 1976). Desde luego, esta no es una postura original de los infrarrealistas sino que el movimiento poético latinoamericano adopta -lo que es un gesto de reconocimiento y de toma de posición - una idea que había sido generada por 
los románticos alemanes y luego implementada por los simbolistas y los movimientos de vanguardia. No creo que Bolaño apunte a modificar el tejido social a tabla rasa en su literatura - Bolaño tiene claro los fracasos del arte en ese sentido-, pero cree que las letras pueden mostrar, exhibir $y$, en algunos casos, presionar los puntos débiles y las heridas abiertas del mundo actual. En este sentido, existe en sus libros la voluntad de dirigir la mirada de sus lectores hasta los aspectos más sórdidos de la sociedad y, por qué no, generar conciencia al respecto.

De esta forma, existen una serie de convergencias entre los infrarrealistas y los románticos alemanes (Novalis, Schiller, Friedich Schlegel). Lo anterior, comienza a quedar de manifiesto al considerar que el romanticismo, más que un movimiento literario, fue "[...] una moral, una erótica y una política. Si no fue una religión fue algo más que una estética: una manera de vivir y una manera de morir" (Paz, 1974: 89). De ahí que Friedrich von Schlegel sostuviera en sus Fragmentos del Athenäum que la poesía romántica no sólo se propone unir todos los géneros en la poesía, contactar la poesía con la filosofía y la retórica, combinar las ideas de belleza, sino que, mediante la acción contradictoria pero convergente de la imaginación y de la ironía, buscaba la fusión entre vida y poesía: “Quiere y debe mezclar poesía y prosa, genialidad y crítica, poesía del arte y poesía de la naturaleza, fundirlas, hacer viva y sociable la poesía y poéticas la vida y la sociedad, poetizar el Witz y llenar y saciar las formas del arte con todo tipo de materiales de creación genuinos, y darles aliento por las vibraciones del humor" (Schlegel, 1974: 137). Para Octavio Paz, los románticos aspiraban a socializar la poesía, desplegando su pensamiento en dos direcciones:

[...] la búsqueda de ese principio anterior que hace de la poesía el fundamento del lenguaje y, por tanto, de la sociedad; y la unión de ese principio con la vida histórica. Si la poesía ha sido el primer lenguaje de los hombres -o si el lenguaje es en su esencia una operación poética que consiste en ver al mundo como un tejido de símbolos y de relaciones entre esos símbolos- cada sociedad está edificada sobre un poema; si la revolución de la edad moderna consiste en el movimiento de regreso de la sociedad a su origen, al pacto primordial de los iguales, esa revolución se confunde con la poesía. (Paz, 1974: 89)

Al igual que los románticos ingleses y alemanes, los movimientos de vanguardia de principios del siglo XX - sobre todo los surrealistas - aspiran a unir vida $y$ arte:

Como el romanticismo, la vanguardia no fue únicamente una estética y un lenguaje; fue una erótica, una política, una visión de 
mundo, una acción: un estilo de vida. La ambición de cambiar la realidad aparece lo mismo en los románticos que en la vanguardia, y en los dos casos se bifurca en direcciones opuestas pero inseparables: la magia y la política, la tentación religiosa y la revolucionaria. (Paz, 1974: 146)

Desde los poetas románticos hasta los surrealistas, los poetas modernos se han enfrentado a esta oposición, sin lograr resolverla ni disolverla. Ciertamente, uno de los casos más interesantes, en este sentido, es el de Rimbaud, quien intenta cambiar la poesía para cambiar la vida:

La alquimia del verbo es un método poético para cambiar a la naturaleza humana; la palabra poética se adelanta al acontecimiento histórico porque es productora o, como él dice (Rimbaud), 'multiplicadora de futuro'; la poesía no sólo provoca nuevos estados psíquicos (como las religiones y las drogas) y libera a los pueblos (como las revoluciones) sino que también tiene por misión inventar un nuevo erotismo y cambiar las relaciones pasionales entre los hombres y las mujeres. Rimbaud proclama que hay que 'reinventar el amor'. (Paz, 1974: 156-157)

En este sentido, es dable apreciar como muchas de las directrices centrales del infrarrealismo ya estaban presentes en movimientos literarios previos. Lo anterior, lejos de convertirse en una simple imitación es, sobre todo, una señal que implica acoger una vertiente de la tradición y, en consecuencia, rechazar otra. Con todo, creo que la relación de Bolaño con los movimientos enunciados supera con creces al deseo de fusionar arte y vida y se extiende, por ejemplo, a la presencia de la alegoría, la ironía, la estética de lo grotesco y lo inusual, entre otros puntos de la producción bolañiana.

Bajo esta perspectiva, el horizonte literario de Bolaño será la hibridez. Su apertura en su propia enunciación de un espacio extranjero que, de pronto, nos hace ver puntos de vista opuestos y diferentes del nuestro (Bhabha, 2002: 5859). Esta hibridez literaria, en un nivel existencial, se relaciona con lo que Foucault definió como "Bio-política" para explicar el particular tipo de experiencia histórica que empieza a producir a partir del alba de la Edad Moderna: "Durante milenios, el hombre siguió siendo lo que era para Aristóteles: un animal viviente y capaz, además de existencia política; el hombre moderno es un animal en cuya política está puesta en entredicho su vida de ser viviente" (Foucault, 1980: 300). La existencia social y política empieza a ser vivida como un lugar donde el hombre se juega su existencia de ser vivo. Experiencia que Enrique Lihn transpone en un verso emblemático, "Porque escribí porque escribí estoy vivo" (Lihn, 1995: 176) que sigue toda la obra 
bolañiana como el más logrado despliegue de la escritura como instante de muerte-vida.

Roberto Bolaño desarrolla una poética que está marcada, entonces, por el romanticismo alemán, el simbolismo y la vanguardia. Es dable sostener que la ruptura y la innovación constituyen dos ejes fundamentales de su propuesta estética. Ahora bien, Roberto Bolaño no solo bebe de la tradición sino que sus obras utilizan la más variada gama de técnicas y recursos literarios para trazar quiebres frente al canon. De allí que, más que abordar la manera en que emergen ecos y reflejos en la literatura de Roberto Bolaño, me interesa establecer como Bolaño subsume la tradición, a fin de alcanzar cierta originalidad. Bolaño asume las influencias de sus precursores y desde de una concepción que siempre consideró a la literatura como una batalla ("La literatura se parece mucho a las peleas de los samuráis", "hay que salir y dar la pelea y darla, además, de la mejor forma posible, de cara y limpiamente, sin pedir cuartel") enfrenta a la tradición para optar a la "pervivencia" literaria.

Harold Bloom plantea que los escritores deben enfrentar a la tradición - uno o varios autores - y subsumirlos para generar una obra propia. Peter de Bolla sostiene que la influencia - en términos de Bloom- es una categoría tropológica, “[...] describe las relaciones entre los textos, es un fenómeno intertextual... tanto la defensa psíquica interna -la experiencia de la angustia por parte del poeta - como las relaciones históricas externas de los textos entre sí son el resultado de una lectura equivocada, o de un encubrimiento poético, y no la causa" (Bloom, 1995: 18). De esta forma, Bloom sostiene que el escritor debe soportar la angustia de las influencias si se desea alcanzar una originalidad significativa dentro de la riqueza de la tradición, la que "[...] no es solo una entrega de testigo o un amable proceso de transmisión: es también una lucha entre el genio anterior y el actual aspirante, en la que el premio es la supervivencia literaria, la inclusión en el canon" (Bloom, 1995: 18). De esta forma, el escritor lee "[...] de una manera errónea $-\mathrm{y}$ creativa-, y por tanto malinterpreta, un texto o textos precursores. Un auténtico escritor canónico puede interiorizar o no la angustia de su obra, pero eso importa poco: la gran obra que consigue escribir es la angustia" (Bloom, 1995: 18). ¿Qué hace posible que los textos de Bolaño sobrevivan en la literatura occidental?

La respuesta, en casi todos los casos, ha resultado ser la extrañeza, una forma de originalidad que o bien no puede ser asimilada o bien nos asimila de tal modo que dejamos de verla como extraña. Walter Pater definió el Romanticismo como la suma de extrañeza y belleza, pero creo que con tal formulación caracterizó no solo a los románticos, sino a toda la escritura canónica. (Bloom, 1995: 13) 
Extrañeza y belleza son, en definitiva, las llaves maestras que han de otorgar a determinados autores un estatus canónico. La fuerza estética (dominio del lenguaje metafórico, originalidad, poder cognitivo, sabiduría y exuberancia en la dicción) es lo que permite irrumpir en el canon occidental.

Bajo esa lógica, se lee, por ejemplo, la obra de Jorge Luis Borges en la literatura de Roberto Bolaño. La literatura de Roberto Bolaño evidencia la marcada influencia de uno de sus máximos precursores, pero distorsionando, a su vez, el proyecto borgiano a fin lograr una voz propia, es decir, Bolaño incluye a la literatura borgiana como un componente de una síntesis más abarcadora. No se puede soslayar, en este sentido, que incluir tiene que ver con considerar algo como parte de un conjunto más amplio. Se trata de un caso particular sometido a un principio en términos de la DRAE. Bolaño incluye a Borges no para imitar su producción literaria sino para que su propia obra crezca con la del autor argentino. Para abordar este punto parto de una afirmación de Banville sobre Borges. Banville afirma que los cuentos de Borges le parecen "[...] carentes de sangre, de sensibilidad" (Corral, 2011: 227) y respecto a Bolaño: "Leí tres o cuatro de sus libros y es auténtico. Desprecia la moda del realismo mágico; él es realista aunque con una mirada distinta, su obra tiene un giro extraño" (Corral, 2011: 227). Hay varios elementos interesantes de esa referencia. Por un lado, el hecho de que Banville rescate el "giro extraño" de la obra de Bolaño. En la lógica de Bloom, constituye una de las exigencias centrales para ingresar en el canon. Por otra parte, las carencias que Banville nota en Borges son implementadas por Bolaño en sus libros, es decir, la "sangre y la sensibilidad". Bolaño adopta la forma lúdica y experimental de la propuesta narrativa borgiana así como su elevada apuesta estética pero llenándola de aquellos elementos despreciados por el autor de Ficciones. De ahí que Bolaño detone profundas cargas eróticas en sus textos, formulando una "anarquía erótica" que lleva hasta el extremo el goce del cuerpo en sus novelas, cuentos y poemas $\mathrm{y}$, especialmente, en Los detectives salvajes. ${ }^{1}$ Asimismo, realiza poderosas exploraciones y revelaciones de mal y la violencia a escala global. ${ }^{2}$ De esta forma, es posible afirmar que la literatura de Roberto Bolaño se transforma, en términos de Deleuze, en una literatura pornológica que apunta hacia la confrontación del lenguaje con sus propios límites, con lo que adquiere el sentido de un no lenguaje donde la violencia y erotismo juegan un papel central. Asimismo, es importante considerar que mientras Borges opta por el

\footnotetext{
${ }^{1}$ Reviso este aspecto de la literatura de Roberto Bolaño in extenso en "Las mil formas de venus en Los detectives salvajes: 'anarquía erótica' en los desiertos de Sonora". Revista Chilena de Literatura, n. 83, pp. 35-60.

${ }^{2}$ Analizo estos elementos de la literatura bolañiana en "Todos los males el mal: La 'estética de la aniquilación' en la narrativa de Roberto Bolaño". Revista Chilena de Literatura n.o 76, pp. 43-70.
} 
relato breve, Bolaño prefiere - a pesar de todas sus declaraciones - el relato extenso, es decir, la novela.

Hay que arrastrar la carga de las influencias si se desea alcanzar una originalidad significativa dentro de la riqueza de la tradición literaria occidental. La tradición no es solo una entrega de testigo o un amable proceso de transmisión: es también una lucha entre el genio anterior y el actual aspirante, en la que el premio es la supervivencia literaria, la inclusión en el canon. (Bloom, 1995: 18)

La malinterpretación errónea y creativa desplegada por Bolaño, al igual que la mezcla de extrañeza y belleza en sus diversas obras, le permite, en mi perspectiva, ingresar al canon chileno, hispanoamericano y occidental. A pesar de lo riesgosa que podría resultar una afirmación de este tipo, me parece que cada vez existe una mayor y mejor evaluación de la obra bolañiana no solo en la crítica literaria hispanoamericana sino a nivel global. En el documental Roberto Bolaño: el último maldito, Mario Vargas Llosa se refirió a la actitud iconoclasta de Bolaño, afirmando que el mito "[...] ha servido en este caso para potenciar el reconocimiento de una obra donde había originalidad y calidad" (Corral, 2011: 14). Además, afirma que es "[...] una literatura difícil, que tiene que educar a sus lectores para ser realmente popular. Ahora ha llegado a un público grande, un público culto" (Corral, 2011: 14). Roberto González Echevarría realiza, por su parte, un comentario muy positivo sobre una de las obras de Bolaño en "Nocturno de Chile y el canon":

[...] creo que Nocturno de Chile permanecerá en el canon de la literatura latinoamericana, y en el de la occidental. Es una pequeña obra maestra al nivel, y a veces por encima del nivel, de lo mejor que escribieron los novelistas consagrados del canon -los del Boom. Pienso que Bolaño es mejor novelista que José Donoso, para ponerlo en un contexto estrictamente chileno. (González Echevarría, 2010: 45)

Si Nocturno de Chile despierta tan positivos comentarios del autor de Mito y Archivo, es posible sostener que las obras mayores de Bolaño - Los detectives salvajes y 2666-, deberían detentar una posición aún más privilegiada en el canon occidental. Es más, el propio Bloom sostuvo en una entrevista que “Roberto Bolaño tenía un talento extraordinario" (Careaga, 2011: 72).

Así, Bolaño dialoga estrechamente con varios autores de la literatura occidental, soportando la angustia de las influencias, para levantar, poco a poco, una extraña y original propuesta literaria. Ante estos antecedentes, es posible sostener, como lo va haciendo de manera cada vez más convincente la crítica 
literaria y algunos de los escritores más relevantes de Occidente, que estamos, probablemente, ante el primer gran autor del siglo XXI.

\section{Bibliografía}

Agamben, Giorgio (2010), Homo Sacer. El poder soberano y la nuda vida. Valencia, Pre-Textos.

Aguilar, Gonzalo (2002), "Roberto Bolaño, entre la historia y la melancolía”, MAnzoni, Celina (ed.), Roberto Bolaño la escritura como tauromaquia. Buenos Aires, Ediciones Corregidor, pp. 145-151.

BHABHA, Homi K. (2002), El lugar de la cultura. Trad. César Aira. Buenos Aires, Manantial.

BLoom, Harold (1995), El canon occidental. Trad. Damián Alou. Barcelona, Editorial Anagrama.

Bolaño, Roberto (1976), "Déjenlo todo, nuevamente. Primer manifiesto infrarrealista". Consultado el 28 de octubre de 2007 en http://www.infrarrealismo.com/ (2007), La universidad desconocida. Barcelona, Anagrama.

BoullosA, Carmen. (2002). "Entrevista a Roberto Bolaño", MAnzonı, Celina (ed.), Roberto Bolaño la escritura como tauromaquia. Buenos Aires, Ediciones Corregidor, pp. 105-113.

CANDIA, Alexis (2011), El "paraíso infernal" en la narrativa de Roberto Bolaño. Santiago, Cuarto Propio.

CAREAGA, Roberto (2011), "Harold Bloom: Lo único que nos queda es la literatura" en La Tercera, Santiago, 8 de octubre, p. 72.

CoRRAL, Wilfrido (2011), Bolaño traducido. Nueva Literatura Mundial. Madrid, Ediciones Escalera.

(2004), "Lo que sobrevivirá del segundo boom Bolaño, el bolañismo", en Revista Quimera, n.ㅇ 241, pp. 32-37.

FouCAULt, Michel (1980), Historia de la sexualidad. La voluntad de saber. Madrid, Siglo XXI.

González Echevarría, Roberto (2010), "Nocturno de Chile y el canon”, en Acta literaria, n.․ 41, pp. 117-128.

LIHN, Enrique (1995), Porque escribí. Santiago, Fondo de Cultura Económica.

MANZONI, Celina (2008), "Ficción de futuro y lucha por el canon en la narrativa de Roberto Bolaño", en Paz Soldán, Edmundo y Faverón Patriau, Gustavo (eds.), Bolaño salvaje. Barcelona, Candaya, pp. 335-357.

PAZ, Octavio (1974), Los hijos del limo. Barcelona, Seix Barral.

SCHLEgEL, Friedrich (1994), Fragmentos del Atheneum. En Fragmentos para una teoría romántica del arte. Madrid, Tecnos. 
VilLALOBOS-RUMINOTT, Sergio (2012), "Literatura y co-pertenencia: Roberto Bolaño y el retorno de la literatura mundial”, en Rodríguez FreIRE, Raúl (ed.), Fuera de Quicio. Bolaño en el tiempo de sus espectros. Santiago, Ripio Ediciones, pp. 193215. 